\title{
The Psychometric Properties of the Center for Epidemiological Studies Depression Scale (CES-D) for Iranian Cancer Patients
}

\author{
Hamid Sharif Nia ${ }^{1}$, Maryam Rezapour ${ }^{2}$, Kelly A. Allen ${ }^{3}$, Saeed Pahlevan Sharif ${ }^{4}$, \\ Azar Jafari ${ }^{5}$, Hojjat Torkmandi ${ }^{6}$, Amir Hossein Goudarzian ${ }^{5 *}$
}

\begin{abstract}
Objectives: The Center for Epidemiologic Studies Depression Scale (CES-D) was specifically created to assess depression in cancer patients. However, to date, the CES-D has not been validated in Farsi. Therefore, this study aimed to assess the psychometric properties of the CES-D in Iranian cancer patients. Methods: During a three-month period (October to December, 2015), a total of 380 cancer patients completed a Farsi version of the CES-D. The construct validity of the scale was evaluated by exploratory factor analysis. Reliability was assessed using Cronbach's alpha and McDonald Omega. All of the statistical procedure were run by SPSS 22 (SPSS Inc., Chicago, IL, USA). Results: The construct validity of the CES-D determined three factors (somatic affect, negative affect, and positive affect), which explained $65.60 \%$ of the total variance. The internal consistency was greater than 0.70 . Conclusion: Findings revealed that the Farsi version of the CES-D has acceptable validity and reliability, which can be used to measure depression in Iranian cancer patients.
\end{abstract}

Keywords: Cancer- depression- epidemiological- psychometrics- Iran

Asian Pac J Cancer Prev, 20 (9), 2803-2809

\section{Introduction}

Cancer is the second most common cause of death in developed and industrialized countries (Ferlay et al., 2015). In 2017, it was estimated that approximately 100 million people had some form of cancer globally that claimed an estimated 1.9 million lives (Institute for Health Metrics and Evaluation, 2017). It has been reported that cancer rates have increased by up to $68 \%$ since the 1990 s and this prevalence is predicted to rise (Edwards et al., 2010). In Iran, the prevalence of cancer increased by 19\% between 2008 and 2012 (Mohebbi et al., 2017). It is predicted that the rates of cancer will multiply in Iran by 2035 (Mohebbi et al., 2017).

Cancer patients can face negative psychological sequelae that can accompany their illness and treatment (Hong and Tian, 2014). Depression is a commonly occurring psychological disorder in patients with cancer which can present as both debilitating and chronic (Hong and Tian, 2014; Aghakhani et al., 2011). Globally, approximately $33 \%$ of cancer patients around the world are suffering from depression, and this trend is also observed in Iran (Arrieta et al., 2013; Jasemi et al., 2016).
Depression has been found to be associated with quality of life (Higginson and Costantini, 2008), increased risk of suicide (Couper et al., 2013), exacerbated suffering (McFarland and Holland, 2018), emotional difficulties and the need for support in family members (Rhondali et al., 2015), increased time in hospital (Smith, 2015), and increased risk of mortality (due to the negative effects during treatment) (Chida et al., 2008; Lutgendorf et al., 2010; Satin et al., 2009).

Notably, the religious and cultural context in which a person lives plays a central role in understanding how people perceive, manage, and respond to depression (Kleinman and Good, 1986). The religious and cultural backgrounds of people have been found to influence a person's experience depression (Sharif Nia et al., 2017). For example, it is widely established that people from Eastern contexts are more likely to emphasize physical effects of depression, whereas people from Western cultures are more likely to emphasize the psychological effects (Ryder et al., 2008). The cultural context for cancer patients may also influence the perceived implications of depression (Davies, 2016). For example, Islamic populations may view depression differently from

${ }^{1}$ School of Nursing and Midwifery Amol, ${ }^{2}$ Psychiatry and Behavioral Sciences Research Center, Addiction Institute, Department of Psychiatry, ${ }^{5}$ Student Research Committee, Mazandaran University of Medical Sciences, Sari, ${ }^{6}$ Operating Room Group, Department of Nursing, School of Nursing and Midwifery, Zanjan University of Medical Sciences, Zanjan, Iran, ${ }^{3}$ The Melbourne Graduate School of Education, the University of Melbourne, Melbourne, Australia, ${ }^{4}$ Taylor's Business School, Taylor's University Malaysia, Subang Jaya, Malaysia.*For Correspondence: amir_sari@yahoo.com 
other cultures where beliefs about death and the afterlife can vary (Sharif Nia et al., 2017). Cultural context is an important consideration in assessing depression, thus it is necessary to develop an accurate tool for Iranian cancer patients and not assume that tools developed in other contexts are appropriate (Gilbert, 2016).

While various measures of depression exist [e.g., Hospital Anxiety and Depression Scale (HADS) (Janda et al., 2017); Beck Depression Inventory (BDI) (Shinn et al., 2017); and Center for Epidemiologic Studies Depression Scale (CES-D) (Siddaway et al., 2017)], the CES-D has been evaluated and used with various populations including patients with cancer (Chen et al., 2010; Crespi et al., 2008; Den Oudsten et al., 2009; Santor et al., 2006).

The CES-D was initially developed and evaluated by Radloff (1977) to study the epidemiology of depressive symptoms in the general population. The scale combined items from previously validated scales, including the Beck Depression Inventory, the Zung Self-Rating Depression Scale, and the Raskin Depression Rating Scale. The CES-D was designed to capture four factors: depressed affect ( 5 items), anhedonia (4 items), somatic complaints (6 items), and interpersonal concerns ( 2 items). It has been adapted and validated in different languages, including Chinese (Wang et al., 2013; Yang et al., 2015; Zhang et al., 2012); Vietnamese (Thanh et al., 2016); and French (Cartierre et al., 2011) and used as a research tool in longitudinal studies (Bakitas et al., 2009; Ghazali et al., 2014). Based on a review of available literature, a Farsi version of the CES-D has not been created and tested. Therefore, the present study aimed to assess the psychometric properties of the CES-D in Iranian cancer patients.

\section{Materials and Methods}

\section{Study design and sample}

During a three-month period (October to December, 2015) a total of 600 patients were admitted to the oncology ward of a university hospital in Sari, Iran. Participants of the study had (i) a current diagnosis of cancer; (ii) were aged 18 years or older; (iii) had not taken antidepressants within the last 6 months; (iv) had the ability to read and write Farsi; (v) demonstrated the capacity to be alert, oriented, and cognitively intact; (vi) had been hospitalized for at least 24 hours or more; (vii) were not being transferred to another hospital; (viii) had no other critical co-occurring conditions (e.g., alcohol or other drug addiction); and (ix) displayed no linguistic and/or physical problems that impeded their abilities to participate in the current study.

The minimum sample size for conducting a factor analysis was determined to be at least 5 to 10 times the number of tested items (Plichta et al., 2013). Out of the 600 participants, 150 patients were excluded based on the inclusion criteria stated above. A total of 52 participants were under the age of 18,71 had taken antidepressants during the last 6 months, and 27 had acute medical conditions. Of the remaining 450 eligible participants, 380 agreed to participate, and they had a response rate of $63.3 \%$.

\section{Data collection and procedures}

Participants were allocated to the study using non-random sampling (i.e., accessible sampling). That is, patients who met the inclusion criteria and had been referred by nursing staff. Informed consent was obtained after participants were informed about the purpose of the study and their right to withdraw at any time. Approximately half of the participants filled out the questionnaire with assistance of an interviewer due to poor eyesight. On average, it took 15 minutes to fill out the self-administered questionnaire.

\section{The CES-D}

The CES-D is used to measure symptoms associated with depression that have been experienced within one week (Vilagut et al., 2016). Each of the 20 items available in this instrument are measured using the Likert scale in the following way: $0=$ rarely or never (less than one day); 1 = occasionally or in few cases (1 to 2 days); $2=$ occasionally or a moderate amount of time ( 3 to 4 days); and $3=$ most of the time or all the time ( 5 to 7 days) (Wang et al., 2013). The total scores range from 0 to 60 . Higher scores indicate greater depressive symptomatology (Maass et al., 2015). The validity and reliability of this scale were proven in various studies (Wang et al., 2013; Yang et al., 2015; Zhang et al., 2012).

\section{Ethical considerations}

This study was approved by the Ethics Committee of Mazandaran University of medical sciences (IR.MAZUMS. REC.95.S.121). Only patients who had agreed and signed the informed consent form participated in this study.

\section{The Verification of the Farsi Version of the CES-D}

For the present study, the verification procedure included a Farsi translation, synthesis, back translation, and expert committee review. Two scholars independently translated the CES-D from English to Farsi. One translator was a professor from a well-known graduate school of nursing, and the other was an associate professor of a graduate institute of long-term care. Both scholars received doctoral degrees from English-speaking countries. During the translation, they recorded comments and ideas when they encountered controversial phrases or uncertainties about wording, and they made annotations accordingly. Two written reports were then completed (Sperber, 2004).

\section{Synthesis}

The two Farsi versions were synthesized into one. The two translators deliberated over each word and item and provided their consent to the final version. The corresponding author of this study integrated the translation after careful consideration, then completed a written report (Sperber, 2004).

\section{Back translation}

Two bilingual scholars without medical backgrounds (Dunbar et al., 2000; Sperber, 2004) translated the synthesized Farsi version back into English. One translator had obtained a degree in linguistics in Malaysia, and the other was a Singaporean graduate student. The main 
purpose of this endeavor was to determine whether the back-translated version and the synthesized Farsi version were the same in terms of the linguistic content and meaning.

\section{Construct validity assessment}

To assess the construct validity, the factor structure of the Farsi version of the CES-D was examined by conducting a maximum likelihood exploratory factor analysis (MLEFA), followed by a Promax rotation with SPSS 22 (SPSS Inc., Chicago, IL, USA). Next, 380 patients were asked to complete the Farsi version of the CES-D. The Kaiser-Meyer-Olkin (KMO) test and Bartlett's test of sphericity were used to check the appropriateness of the study sample and the factor analysis model. The number of latent factors was estimated by using parallel analysis. The items with absolute loading values of 0.3 or greater were regarded as appropriate (Saggino and Kline, 1996).

\section{Reliability assessment}

The reliability of the Farsi version of the CES-D was first assessed by evaluating its internal consistency and calculating the Cronbach's alpha $(\alpha)$ and McDonald's omega $(\Omega)$ (Javali and Gudaganavar, 2011). A reliability of 0.7 or greater showed a satisfactory internal consistency (Jorritsma et al., 2012). Intra-class correlation coefficients (ICC) were used to establish the test-retest reliability of the CES-D over an interval of two weeks by using a two-way mixed ICC that showed an absolute agreement at the level of individual items. The results were interpreted as follows: $0-0.2=$ low; $0.21-0.40=$ fair; $0.41-0.60=$ moderate; $0.61-0.80=$ substantial; and $0.81-1=$ almost perfect (Landis and Koch, 1977).

\section{Multivariate normality and outliers}

Univariate distributions were examined for outliers, skewness, and kurtosis. Multivariate distributions were evaluated for normality and multivariate outliers (Sharif Nia et al., 2017). Multivariate normality can be evaluated through the use of the Mardia's coefficient of multivariate kurtosis. One indication of deviation from normal distribution was a Mardia's coefficient greater than 8 (Raoprasert and Islam, 2010). Multivariate outliers were evaluated through the evaluation of a Mahalanobis distance (Harrington, 2008). Items with a Mahalanobis distance of $\mathrm{p}<.001$ were considered to be multivariate outliers (Tabachnick and Fidell, 2013).

\section{Results}

Preliminary analyses and descriptive information

A demographic profile of 380 cancer patients is summarized in Table 1. Generally, male patients (48.39 \pm $13 \pm 39 ; 95 \%$ CI: 46.41-50.38) were older than the females (45.33 $\pm 18.44 ; 95 \%$ CI: 42.79-47.87).

\section{Construct Validity}

The KMO was 0.911, and the Bartlett's test of sphericity was significant $(\mathrm{P}<0.001)$, which indicated that the sampling was adequate. Table 2 shows the results of MLEFA on the Farsi Version of the CES-D. The MLEFA revealed that three combined factors accounted for 65.60 $\%$ of the variance. Factor 1 (somatic affect) had 7 items. Factor 2 (negative affect) had 5 items, and Factor 3

Table 1. Demographic Profile of Participants

\begin{tabular}{lccc}
\hline Characteristic & $\mathrm{N}(\%)$ & Characteristic & $\mathrm{N}(\%)$ \\
\hline Sex & & Family history of cancer & $112(29.5)$ \\
Male & $175(46.1)$ & Yes & $268(70.5)$ \\
Female & $205(53.9)$ & No & $261(68.7)$ \\
Economic status & & Depression & $119(31.3)$ \\
Weak & $110(28.9)$ & Down & $146(38.42)$ \\
Average & $204(53.7)$ & Up & $57(15)$ \\
Good & $66(17.4)$ & Past medical history* & $141(37.1)$ \\
Education & & Cardiac diseases & $36(9.48)$ \\
Illiterate & $210(55.3)$ & Respiratory diseases & \\
Diploma & $138(36.3)$ & Gastric diseases & $71(18.7)$ \\
BS & $22(5.8)$ & Urinary diseases & $309(81.3)$ \\
MSs and above & $10(2.6)$ & History of cigarette smoking & Mean (SD) \\
Marital status & & Yes & $46.74(16.328)$ \\
Single & $51(13.4)$ & No & \\
Married & $329(86.6)$ & Characteristic & \\
Cancer stage & $132(34.7)$ & Age & \\
One & $133(35)$ & & \\
Two & $92(24.2)$ & & \\
Tree & $23(6.1)$ & & \\
Four & & & \\
\hline
\end{tabular}

* Number of patients who had these diseases 
Table 2. Factor Analysis for the Persian Version of CES-D in Patients with Cancer

\begin{tabular}{|c|c|c|c|c|c|c|c|}
\hline Factors & Factors name & Items & Loading & $\mathrm{h}^{2}$ & $\%$ of Variance & Eigenvalues & Internal consistency \\
\hline \multirow{7}{*}{1} & \multirow{7}{*}{ Somatic } & $\begin{array}{l}\text { Q1. I was bothered by things that usually } \\
\text { don't bother me. }\end{array}$ & 0.922 & 0.803 & \multirow{7}{*}{32.121} & \multirow{7}{*}{5.622} & \multirow{7}{*}{$\alpha=.916, \Omega=.953$} \\
\hline & & Q2. I did not feel like eating: my appetite was poor. & 0.875 & 0.755 & & & \\
\hline & & Q3. I felt that everything I did was an effort. & 0.801 & 0.721 & & & \\
\hline & & Q11. My sleep was restless. & 0.786 & 0.533 & & & \\
\hline & & Q20. I could not get "going." & 0.762 & 0.672 & & & \\
\hline & & $\begin{array}{l}\text { Q5. I had trouble keeping my mind on what } \\
\text { I was doing. }\end{array}$ & 0.681 & 0.597 & & & \\
\hline & & Q13. I talked less than usual. & 0.597 & 0.642 & & & \\
\hline \multirow{5}{*}{2} & \multirow{5}{*}{ Negative affect } & $\begin{array}{l}\text { Q12. I felt that I could not shake off the blues, even } \\
\text { with the help from family or friends. }\end{array}$ & 0.824 & 0.592 & \multirow{5}{*}{18.765} & \multirow{5}{*}{3.620} & \multirow{5}{*}{$\alpha=.891, \Omega=.885$} \\
\hline & & Q6. I felt depressed. & 0.722 & 0.634 & & & \\
\hline & & Q14. I felt lonely. & 0.691 & 0.533 & & & \\
\hline & & Q17. I had crying spells. & 0.655 & 0.683 & & & \\
\hline & & Q18. I felt sad. & 0.583 & 0.423 & & & \\
\hline \multirow{4}{*}{3} & \multirow{4}{*}{ Positive affect } & Q4. I felt that I was just as good as other people. & 0.819 & 0.637 & \multirow{4}{*}{14.715} & \multirow{4}{*}{2.510} & \multirow{4}{*}{$\alpha=.822, \Omega=.854$} \\
\hline & & Q8. I felt hopeful about the future. & 0.726 & 0.544 & & & \\
\hline & & Q12. I was happy. & 0.654 & 0.613 & & & \\
\hline & & Q16. I enjoyed life. & 0.598 & 0.413 & & & \\
\hline
\end{tabular}

Abbreviation, $\mathrm{h}^{2}$, Communalities

(positive affect) had 4 items.

\section{Reliability}

As reported in Table 2, the Cronbach's alpha and McDonald's omega demonstrated good reliability and internal consistency for three factors. The average ICC was 0.841 with a $95 \%$ confidence interval from 0.703 to $0.901(\mathrm{p}<.001)$.

\section{Discussion}

The results of the present study supported a three-factor structure: somatic symptoms, negative affect, and positive affect for the CES-D scale. These three factors explained $65.60 \%$ of the variance.

Thanh et al. (2016) reported that the CES-D scale consisted of two factors (negative affect and positive affect). A three-factor structure of the CES-D has been reported previously in a sample of Arab females (interpersonal problems, somatic symptoms, and positive affect) (Ghubash et al., 2000). Zhang et al., (2012) also presented a three factor model: positive affect, interpersonal problems, and a combination of depressive mood and somatic symptoms of the CES-D scale in a study in rural China. Also, a three-factor structure of the CES-D has been identified by Fountoulakis et al., (2001), which included positive affect, a combination of irritability and problems with interpersonal relationships, and a combination of depressive symptoms and somatic symptoms.

However, other research has reported four factors. Zhang et al., (2015), Thombs et al., (2008), and Chin et al., (2015) revealed four factors of the CES-D scale (depressed affect, somatic symptoms, positive affect, and interpersonal problems) Hair et al., (2010) stated that in the psychological studies and human sciences, the extraction of factors is appropriate when the explained variance falls between $50 \%$ and $60 \%$.

The present study differs from previous research in terms of the number of factors identified and the participant cohort and context. The three-factor structure of the CES-D was identified in the present study, but a two-factor structure of it (Kwakkenbos et al., 2013; Thanh et al., 2016) and four-factor structure of it (Chin et al., 2015; Thombs et al., 2008; Zhang et al., 2015) were reported in other studies (Ghubash et al., 2000; Zhang et al., 2012).

The first factor identified in our study was somatic affect. Somatic affect can related to fatigue, loss of energy, and physical dysfunctions, such as a disturbance of sleep and appetite (Kapfhammer, 2006). Researchers have found that increased somatic complaints are associated with the onset of depression (Penninx et al., 2013). Also, it seems that individuals with greater somatic affects related depression indicate a reduced awareness of behavioral errors (Bridwell et al., 2015; Northouse et al., 2010). This factor was supported by past research (Chin et al., 2015; Fountoulakis et al., 2001; Ghubash et al., 2000; Thombs et al., 2008; Zhang et al., 2012; Zhang et al., 2015).

The second factor of the CES-D scale found in the study was negative affect. Negative affect usually refers to feelings of sadness and worthlessness (Olino et al., 2011). Individuals with depressive disorders experience a lower positive affect, higher negative affect, and more self-blame after stressful events (Compton et al., 2013). This factor was also identified in the study of Thanh et al., (2016) and Kwakkenbos et al., (2013).

The third factor identified in the present study was positive affect. This affect plays an important adaptive role in physical and psychological health, and it can prevent depression as a protective factor (Jaser et al., 2011). Positive affect is associated with adaptability and flexible thinking (Danhauer et al., 2013). On the other hand, a reduced positive affect can predict the onset of 
depression (Nelis et al., 2015). In line with this study, this factor appeared in several other studies as an important aspect of the CES-D instrument (Chin et al., 2015; Fountoulakis et al., 2001; Ghubash et al., 2000; Thombs et al., 2008; Zhang et al., 2012).

According to the findings of this study, the coefficients of internal consistency for the overall CES-D scale indicated that this scale had an acceptable reliability. Also, the reliability of this scale has been assessed by Cronbach's alpha in several other studies (Lehmann et al., 2011; Makambi et al., 2009; Zhang et al., 2015). For instance, the internal consistency (Cronbach's $\alpha$ ) of the CES-D scale was 0.85 , with a test-retest correlation coefficient (r) of 0.64 in the study conducted by Zhang et al., (2015). Also, Makambi et al., (2009) tested the internal consistency of data by Cronbach's $\alpha$ coefficient and split-half coefficient, which yielded a magnitude of over 0.85 for both indices.

\section{Limitations}

The researchers of this study have ensured that the forward-backward translation method was performed at a high standard, and the original author of the scale confirmed the accuracy of the translation. Apart from this confirmation, there's always the potential of using a scale that was originally designed for a different population. Cultural differences and language nuances may not be translatable, and test users would be advised to remain cognizant about this potential issue. Also, when the researchers of this study completed questionnaires for patients with vision difficulties, they could have reported biased responses.

\section{Recommendations}

Future validation studies with samples from different populations (as well as longitudinal designs) are suggested to verify the findings of this study. Also, since Iranian populations reside all over the world, it could beneficial to test the tool in Iranians in Europe, Asia, Australia, and the USA in order to determine its generalizability for all Iranian populations.

\section{Nursing Implication}

The Farsi Version of the CES-D can be used by nurses and other hospital staff members in cancer units to screen for symptoms of depression. Also, it can be used in nursing education as a component of the nursing process (including diagnosis). Finally, using a valid, reliable tool in nursing research can demonstrate valuable results that researchers can use in cancer patients.

In conclusion, this study confirmed acceptable psychometric properties, as well as the factor structure of the CES-D in an Iranian sample. Given these findings, the scale can be used as a valid, reliable tool for the assessment of depression that is experienced by Iranian cancer patients.

\section{Acknowledgements}

We thank all of the patients that contributed to this research. Also, we are grateful to the research deputy of the Department of Medical Sciences at Mazandaran University for his support.

\section{Conflict of Interest}

The authors declare that there is no conflict of interest about the publication of this article.

\section{References}

Aghakhani N, Sharif Nia H, Eghtedar S, et al (2011). Prevalence of depression among students of urmia university of medical sciences (iran). Iran J Psychiatry Behav Sci, 5, 131-5.

Arrieta Ó, Angulo LP, Núñez-Valencia C, et al (2013). Association of depression and anxiety on quality of life, treatment adherence, and prognosis in patients with advanced non-small cell lung cancer. Ann Surg Oncol, 20, 1941-8.

Bakitas M, Lyons KD, Hegel MT, et al (2009). Effects of a palliative care intervention on clinical outcomes in patients with advanced cancer: the Project ENABLE II randomized controlled trial. Jama, 302, 741-9.

Bridwell DA, Steele VR, Maurer JM, et al (2015). The relationship between somatic and cognitive-affective depression symptoms and error-related ERPs. J Affect Disord, 172, 89-95.

Cartierre N, Coulon N, Demerval R (2011). Confirmatory factor analysis of the short French version of the Center for Epidemiological Studies of Depression Scale (CES-D10) in adolescents. L'Encephale, 37, 273-7.

Chen X, Lu W, Zheng Y, et al (2010). Exercise, tea consumption, and depression among breast cancer survivors. J Clin Oncol, 28, 991-8.

Chida Y, Hamer M, Wardle J, et al (2008). Do stress-related psychosocial factors contribute to cancer incidence and survival? Nature Reviews. Clin Oncol, 5, 466-75.

Chin WY, Choi EP, Chan KT, et al (2015). The psychometric properties of the Center for Epidemiologic Studies Depression Scale in Chinese primary care patients: factor structure, construct validity, reliability, sensitivity and responsiveness. PLos One, 10, 0135131.

Compton RJ, Hofheimer J, Kazinka R, et al (2013). Alpha suppression following performance errors is correlated with depression, affect, and coping behaviors. Emotion, 13, 905-14.

Couper JW, Pollard AC, Clifton DA (2013). Depression and cancer. Med J Aust, 199, S13-6.

Crespi CM, Ganz PA, Petersen L, et al (2008). Refinement and psychometric evaluation of the impact of cancer scale. J Natl Cancer Inst, 100, 1530-41.

Danhauer SC, Legault C, Bandos H, et al (2013). Positive and negative affect, depression, and cognitive processes in the Cognition in the Study of Tamoxifen and Raloxifene (CoSTAR) Trial. Neuropsychol Dev Cogn B Aging Neuropsychol Cogn, 20, 532-52.

Davies N (2016). Culture and Depression: A Cross-Cultural Meta-Analysis of the Zung Self-Rating Depression Scale.

Den Oudsten BL, Van Heck GL, Van der Steeg AF, et al (2009). Predictors of depressive symptoms 12 months after surgical treatment of early-stage breast cancer. Psycho-Oncology, 18, 1230-7.

Dunbar MJ, Robertsson O, Ryd L, et al (2000). Translation and validation of the Oxford-12 item knee score for use in Sweden. Acta Orthop Scand, 71, 268-74.

Edwards BK, Ward E, Kohler BA, et al (2010). Annual report to the nation on the status of cancer, 1975-2006, featuring colorectal cancer trends and impact of interventions (risk 
factors, screening, and treatment) to reduce future rates. Cancer, 116, 544-73.

Institute for Health Metrics and Evaluation. 2017. Global Health Data Exchange [Online]. Available: http://ghdx.healthdata. org/gbd-results-tool.

Ferlay J, Soerjomataram I, Dikshit R, et al (2015). Cancer incidence and mortality worldwide: sources, methods and major patterns in GLOBOCAN 2012. Int J Cancer, 136, Page????

Fountoulakis K, Iacovides A, Kleanthous S, et al (2001). Reliability, Validity and Psychometric Properties of the Greek Translation of the Center for Epidemiological StudiesDepression (CES-D) Scale. BMC Psychiatry, 1, 3.

Ghazali SR, Elklit A, Balang RV, et al (2014). Determining the cut-off score for a Malay language version of the Centre for Epidemiologic Studies Depression Scale (CESD). ASEAN J Psych, 15, 146-52.

Ghubash R, Daradkeh TK, Al Naseri KS, et al (2000). The performance of the Center for Epidemiologic Study Depression Scale (CES-D) in an Arab female community. Int J Soc Psychiatry, 46, 241-9.

Gilbert P 2016. Depression: The evolution of powerlessness, Routledge.

Hair JF, Black WC, Babin BJ, et al 2010. Multivarite Data Analysis. 7th ed, Upper Saddle River, NJ, Prentice Hall.

Harrington D 2008. Confirmatory Factor Analysis, Oxford University Press, USA.

Higginson IJ, Costantini M (2008). Dying with cancer, living well with advanced cancer. Eur J Cancer, 44, 1414-24.

Hong JS, Tian J (2014). Prevalence of anxiety and depression and their risk factors in Chinese cancer patients. Support Care Cancer, 22, 453-9.

Janda M, Neale RE, Klein K, et al (2017). Anxiety, depression and quality of life in people with pancreatic cancer and their carers. Pancreatology, 17, 321-7.

Jasemi M, Aazami S, Zabihi RE (2016). The effects of music therapy on anxiety and depression of cancer patients. Indian $J$ Palliat Care, 22, 455-8.

Jaser SS, Champion JE, Dharamsi KR, et al (2011). Coping and Positive Affect in Adolescents of Mothers With and Without a History of Depression. J Child Fam Stud, 20, 353-60.

Javali SB, Gudaganavar NV (2011). Effect of varying sample size in estimation of reliability coefficients of internal consistency. Webmed Central Biostatistics, 2, page???

Jorritsma W, de Vries GE, Dijkstra PU, et al (2012). Neck pain and disability scale and neck disability index: validity of Dutch language versions. Eur Spine J, 21, 93-100.

Kapfhammer HP (2006). Somatic symptoms in depression. Dialogues Clin Neurosci, 8, 227.

Kleinman A, Good B 1986. Culture and depression: Studies in the anthropology and cross-cultural psychiatry of affect and disorder, Univ of California Press.

Kwakkenbos L, Arthurs E, van den Hoogen FH, et al (2013). Cross-language measurement equivalence of the Center for Epidemiologic Studies Depression (CES-D) scale in systemic sclerosis: A comparison of Canadian and Dutch patients. PLos One, 8, 53923.

Landis JR, Koch GG (1977). The measurement of observer agreement for categorical data. Biometrics, 33, 159-74.

Lehmann V, Makine C, Karşıdağ C, et al (2011). Validation of the turkish version of the centre for epidemiologic studies depression scale (ces-d) inpatients with type 2 diabetes mellitus. BMC Med Res Methodol, 11, 109.

Lutgendorf SK, Sood AK, Antoni MH (2010). Host factors and cancer progression: biobehavioral signaling pathways and interventions. J Clin Oncol, 28, 4094-9.

Maass SW, Roorda C, Berendsen AJ, et al (2015). The prevalence of long-term symptoms of depression and anxiety after breast cancer treatment: a systematic review. Maturitas, 82, 100-8.

Makambi KH, Williams CD, Taylor TR, et al (2009). An assessment of the CES-D scale factor structure in black women: The Black Women's Health Study. Psychiatry Res, 168, 163-70.

McFarland DC, Holland J (2018). Depression and Cancer. Depression As a Systemic Illness, 97.

Mohebbi E, Nahvijou A, Hadji M, et al (2017). Iran Cancer Statistics in 2012 and projection of cancer incidence by 2035. Basic and Clinical Cancer Research, 9, 3-22.

Nelis S, Holmes EA, Raes F (2015). Response Styles to Positive Affect and Depression: Concurrent and Prospective Associations in a Community Sample. Cogn Ther Res, 39, 480-91.

Northouse LL, Katapodi MC, Song L, et al (2010). Interventions with family caregivers of cancer patients: meta-analysis of randomized trials. CA Cancer J Clin, 60, 317-39.

Olino TM, Lopez-Duran NL, Kovacs M, et al (2011). Developmental trajectories of positive and negative affect in children at high and low familial risk for depressive disorder. J Child Psychol Psychiatry, 52, 792-9.

Penninx BW, Milaneschi Y, Lamers F, et al (2013). Understanding the somatic consequences of depression: biological mechanisms and the role of depression symptom profile. BMC medicine, 11, 129.

Plichta SB, Kelvin EA, Munro BH 2013. Munro's statistical methods for health care research, Wolters Kluwer Health/ Lippincott Williams and Wilkins.

Radloff LS (1977). The CES-D scale: A self-report depression scale for research in the general population. Appl Psychol Meas, 1, 385-401.

Raoprasert T, Islam SMN 2010. Designing an Efficient Management System: Modeling of Convergence Factors Exemplified by the Case of Japanese Businesses in Thailand, Physica-Verlag HD.

Rhondali W, Chirac A, Laurent A, et al (2015). Family caregivers' perceptions of depression in patients with advanced cancer: A qualitative study. Palliat Support Care, 13, 443-50.

Ryder AG, Yang J, Zhu X, et al (2008). The cultural shaping of depression: somatic symptoms in China, psychological symptoms in North America? J Abnorm Psychol, 117, 300-13.

Saggino A, Kline P (1996). Item factor analysis of the Italian version of the Death Anxiety Scale. J Clin Psychol, 52, 329-33.

Santor DA, Gregus M, Welch A (2006). FOCUS ARTICLE: Eight decades of measurement in depression. Measurement, 4, 135-55.

Satin JR, Linden W, Phillips MJ (2009). Depression as a predictor of disease progression and mortality in cancer patients. Cancer, 115, 5349-61.

Sharif Nia H, Pahlevan Sharif S, Lehto RH, et al (2017a). Development and psychometric evaluation of a Persian version of the Death Depression Scale-Revised: a crosscultural adaptation for patients with advanced cancer. Jpn J Clin Oncol, 47, 713-9.

Sharif Nia H, Sharif SP, Esmaeili R, et al (2017b). Factors Influencing the Level of Death Depression in Patients with Cancer: A Path Analysis. JMUMS, 26, 318-31.

Shinn EH, Valentine A, Baum G, et al (2017). Comparison of four brief depression screening instruments in ovarian cancer patients: Diagnostic accuracy using traditional versus alternative cutpoints. Gynecol Oncol, 145, 562-8.

Siddaway AP, Wood AM, Taylor PJ (2017). The Center for Epidemiologic Studies-Depression (CES-D) scale measures a continuum from well-being to depression: Testing two key 
predictions of positive clinical psychology. $J$ Affect Disord,

213, 180-6.

Smith HR (2015). Depression in cancer patients: Pathogenesis, implications and treatment. Oncol Lett, 9, 1509-14.

Sperber AD (2004). Translation and validation of study instruments for cross-cultural research. Gastroenterology, 126, S124-S8.

Tabachnick BG, Fidell LS 2013. Using Multivariate Statistics, Pearson Education.

Thanh ND, Quyen BT, Tien TQ (2016). Validation of a Brief CES-D Scale for Measuring Depression and Its Associated Predictors among Adolescents in Chi Linh, Hai Duong, Vietnam. AIMS Public Health, 3, 448-59.

Thombs BD, Hudson M, Schieir O, et al (2008). Reliability and validity of the center for epidemiologic studies depression scale in patients with systemic sclerosis. Arthritis Rheum, $\mathbf{5 9}, 438-43$.

Vilagut G, Forero CG, Barbaglia G, et al (2016). Screening for Depression in the General Population with the Center for Epidemiologic Studies Depression (CES-D): A Systematic Review with Meta-Analysis. PLos One, 11, 0155431.

Wang M, Armour C, Wu Y, et al (2013). Factor structure of the CES-D and measurement invariance across gender in mainland Chinese adolescents. J Clin Psychol, 69, 966-79.

Yang L, Jia C-X, Qin P (2015). Reliability and validity of the Center for Epidemiologic Studies Depression Scale (CES-D) among suicide attempters and comparison residents in rural China. BMC psychiatry, 15, 76.

Zhang J, Sun W, Kong Y, et al (2012). Reliability and validity of the Center for Epidemiological Studies Depression Scale in 2 special adult samples from rural China. Comprehensive psychiatry, 53, 1243-51.

Zhang Y, Ting RZ, Lam MH, et al (2015). Measuring depression with CES-D in Chinese patients with type 2 diabetes: the validity and its comparison to PHQ-9. BMC psychiatry, 15, 198.

\section{(ब) $(1)$}

This work is licensed under a Creative Commons AttributionNon Commercial 4.0 International License. 